\title{
THE EQUATIONS OF REES ALGEBRAS OF EQUIMULTIPLE IDEALS OF DEVIATION ONE
}

\author{
FERRAN MUINOOS AND FRANCESC PLANAS-VILANOVA
}

(Communicated by Irena Peeva)

\begin{abstract}
We describe the equations of the Rees algebra $\mathbf{R}(I)$ of an equimultiple ideal $I$ of deviation one provided that $I$ has a reduction generated by a regular sequence $x_{1}, \ldots, x_{s}$ such that the initial forms $x_{1}^{*}, \ldots, x_{s-1}^{*}$ are a regular sequence in the associated graded ring. In particular, we prove that there is a single equation of maximum degree in a minimal generating set of the equations of $\mathbf{R}(I)$, which recovers some previous known results.
\end{abstract}

\section{INTRODUCTION}

Let $(R, \mathfrak{m})$ be a Noetherian local ring and let $I$ be an equimultiple ideal of $R$ of deviation $\mu(I)-\operatorname{height}(I)=1$. The aim of this paper is to study the equations of $\mathbf{R}(I)=R[I t]=\bigoplus_{n>0} I^{n}$, the Rees algebra of $I$. We will assume that $x_{1}, \ldots, x_{s}, y$ is a minimal generating set of $I$, that $J=\left(x_{1}, \ldots, x_{s}\right)$ is a reduction of $I$ generated by an $R$-sequence and that the initial forms $x_{1}^{*}, \ldots, x_{s-1}^{*}$ in $\mathbf{G}(I)=\bigoplus_{n \geq 0} I^{n} / I^{n+1}$, the associated graded ring of $I$, are a $\mathbf{G}(I)$-sequence.

Recall that the reduction number of $I$ with respect to $J$ is the least integer $r \geq 0$ such that $I^{r+1}=J I^{r}$, denoted by $\mathrm{r}_{J}(I)$ (notice that, with our assumptions, $r \geq 1$ ). Set $V=R\left[X_{1}, \ldots, X_{s}, Y\right]$ and let $\varphi: V \rightarrow \mathbf{R}(I)$ be the polynomial presentation of $\mathbf{R}(I)$ sending $X_{i}$ to $x_{i} t$ and $Y$ to $y t$. Let $Q=\bigoplus_{n>1} Q_{n}$ be the kernel of $\varphi$, whose elements will be referred to as the equations of $\mathbf{R}(I)$. For instance, for $r=\mathrm{r}_{J}(I)$, the containment of $y^{r+1}$ in $J I^{r}$ induces an equation $Y^{r+1}-\sum X_{i} F_{i} \in Q_{r+1}$, with $F_{i} \in V_{r}$. Given an integer $n \geq 1$, set $Q\langle n\rangle \subset Q$, the ideal generated by the homogeneous equations of $\mathbf{R}(I)$ of degree at most $n$ in $X_{1}, \ldots, X_{s}, Y$. The relation type of $I$, denoted by $\operatorname{rt}(I)$, is the least integer $N \geq 1$ such that $Q=Q\langle N\rangle$. When $Q=Q\langle 1\rangle, I$ is said to be of linear type. Our main result is the following.

Theorem 4.1. Let $(R, \mathfrak{m})$ be a Noetherian local ring and let $I$ be an ideal of $R$. Let $x_{1}, \ldots, x_{s}, y$ be a minimal generating set of $I$ and $J=\left(x_{1}, \ldots, x_{s}\right)$. Assume that $x_{1}, \ldots, x_{s}$ is an $R$-sequence and that $x_{1}^{*}, \ldots, x_{s-1}^{*}$ is a $\mathbf{G}(I)$-sequence. Then, for each $n \geq 2$, the map sending $F \in Q_{n}$ to $F(0, \ldots, 0,1) \in\left(J I^{n-1}: y^{n}\right)$ induces an isomorphism of $R$-modules

$$
\left[\frac{Q}{Q\langle n-1\rangle}\right]_{n} \cong \frac{J I^{n-1}: y^{n}}{J I^{n-2}: y^{n-1}}
$$

Received by the editors March 16, 2011 and, in revised form, August 22, 2011.

2010 Mathematics Subject Classification. Primary 13A30.

Key words and phrases. Blowing-up algebras, relation type, reduction number.

The second author is partially supported by the Spanish grant MTM2010-20279. 
In particular, if $J$ is a reduction of $I$ with reduction number $r=r_{J}(I)$, then $r t(I)=$ $r_{J}(I)+1$ and there is a form $Y^{r+1}-\sum X_{i} F_{i} \in Q_{r+1}$, with $F_{i} \in V_{r}$, such that $Q=\left(Y^{r+1}-\sum X_{i} F_{i}\right)+Q\langle r\rangle$.

Note that, with these assumptions, $s \leq \operatorname{grade}(I) \leq \operatorname{height}(I) \leq l(I) \leq \mu(J)=$ $s$, where $l(\cdot)$ and $\mu(\cdot)$ stand for the analytic spread and the minimal number of generators, respectively. Thus $I$ is an equimultiple ideal (i.e., height $(I)=l(I)$ ) of deviation $\mu(I)-$ height $(I)=1$. Roughly speaking, the theorem says how to obtain a minimal generating set of the equations of $\mathbf{R}(I)$. For the equations of degree 1: pick a minimal generating set of the first syzygies of $I$, viewed as elements of $Q_{1}$; for the equations of higher degree $n, 2 \leq n \leq r+1$, take representatives of the inverse images of a minimal generating set of $\left(J I^{n-1}: y^{n}\right) /\left(J I^{n-2}: y^{n-1}\right)$ (see Remark 2.2 and Example 5.1).

Thus far, the study of the equations of $\mathbf{R}(I)$ has produced a vast literature. Part of this work has been focused on ideals having small deviations as well as on the interplay between the reduction number and the relation type (see, just to mention a few of them, 12, [13, [14], 15], 23, 24], 26], 27]). The particular hypotheses and interests in this note owe much to the works of T. Cortadellas and S. Zarzuela in [3, W. Heinzer and M.-K. Kim in [7, S. Huckaba in [12] and [13], N.V. Trung in [23] and 24], and W.V. Vasconcelos in [26]. In fact, Theorem 4.1 sprouted as an attempt to understand [26, Theorem 2.3.3]. That is one of the main reasons for considering ideals $I$ of the form $I=(J, y), J$ being a reduction of $I$ generated by a sufficiently good sequence. The reader may also consult [10], 11] for a recent account on the equations of $\mathbf{R}(I)$ with similar assumptions.

Even in a simpler case, i.e. $\mu(I)$ - height $(I)=0$, the equations of $\mathbf{R}(I)$ may be difficult to describe. Remarkably, any ideal $I$ of the principal class is generated by an $R$-sequence $x_{1}, \ldots, x_{s}$, provided that $I$ is prime (4]) or $R$ is Cohen-Macaulay. In both cases the equations are generated by the Koszul relations $x_{i} X_{j}-x_{j} X_{i}$, $1 \leq i<j \leq s$. In particular, $I$ is of linear type. However, if $I$ is not prime and $R$ is not Cohen-Macaulay, this is no longer true. For instance, consider the ideals generated by a system of parameters. C. Huneke asked in [16] whether there is a uniform bound for the relation type of these ideals in a complete local equidimensional Noetherian ring $R$. The full answer to this question was given in [28, [19] and [1]. Concretely, in [1, Example 2.1], it was shown that if the non-Cohen-Macaulay locus of $R$ has dimension 2 or more, there exist families of parametric ideals of $R$ with unbounded relation type. This gives an idea of the complexity of the structure of the equations of $\mathbf{R}(I)$.

In our case, i.e. $\mu(I)$ - height $(I)=1$, if $R$ is Cohen-Macaulay, one has that $I$ is of linear type if and only if $I$ is locally of the principal class at all minimal primes of $I$ (see [9, Theorem 4.8]). But, if $I$ is not locally of the principal class at its minimal primes, then the relation type may be arbitrarily large. For instance, take $R=k\left[\left[a_{1}, a_{2}\right]\right]$, the power series ring in two variables $a_{1}, a_{2}$ over a field $k$, set $x_{1}=$ $a_{1}^{p}, x_{2}=a_{2}^{p}$ and $y=a_{1} a_{2}^{p-1}$, with $p \geq 2$. Let $I=\left(x_{1}, x_{2}, y\right)$. Then $I$ has deviation one and is $\left(a_{1}, a_{2}\right)$-primary, hence not locally of the principal class at its unique minimal prime. One can check that $\operatorname{rt}(I) \geq p$. In fact, $I$ fulfills the hypotheses of Theorem 4.1, $J=\left(x_{1}, x_{2}\right)$ being a reduction of $I$. Thus, the containment $y^{p} \in$ $J I^{p-1}$ induces the only equation of degree $p$ in a minimal generating set of equations of $\mathbf{R}(I)$ and $\operatorname{rt}(I)=p$ (see Example 5.1). 
As said before, this note has its origins in the following result of W.V. Vasconcelos in [26, Theorem 2.3.3]: let $(R, \mathfrak{m})$ be a Cohen-Macaulay local ring of dimension $d$ and let $I$ be an $\mathfrak{m}$-primary ideal of $R$. Let $x_{1}, \ldots, x_{d}, y$ be a minimal generating set of $I$, where $J=\left(x_{1}, \ldots, x_{d}\right)$ is a reduction of $R$ with reduction number $\mathrm{r}_{J}(I)=1$. Then there is a form $Y^{2}-\sum X_{i} F_{i} \in Q_{2}$, with $F_{i} \in V_{1}$, such that $Q=\left(Y^{2}-\sum X_{i} F_{i}\right)+Q\langle 1\rangle$. In particular, $\operatorname{rt}(I)=2$.

Observe that the hypotheses of [26, Theorem 2.3.3] imply that $x_{1}, \ldots, x_{d}$ is an $R$ sequence and that the initial forms $x_{1}^{*}, \ldots, x_{d}^{*}$ are a $\mathbf{G}(I)$-sequence (see the result of P. Valabrega and G. Valla in [25, Proposition 3.1]). By Theorem 4.1] it is enough to suppose that $x_{1}^{*}, \ldots, x_{d-1}^{*}$ is a $\mathbf{G}(I)$-sequence and to consider any reduction number (see Corollary 4.5).

Also as a corollary of Theorem 4.1, we recover the result of W. Heinzer and M.-K. Kim in [7, Theorem 5.6], where they prove that the equations of $\mathbf{F}(I)=$ $\mathbf{R}(I) \otimes R / \mathfrak{m}=\bigoplus_{n>0} I^{n} / \mathfrak{m} I^{n}$, the fiber cone of $I$, are generated by a unique equation of degree $\mathrm{r}_{J}(I)+1$.

The paper is organised as follows: in Section 2 we recall from [20] how to express the equations of a standard algebra in terms of the effective relations and the Koszul homology. Section 3 is focused on the equations of the Rees algebra. In Section 4 we prove Theorem 4.1 and the aforementioned results of W. V. Vasconcelos and W. Heinzer and M.-K. Kim. We finish by giving some examples in Section 5 ,

All the unexplained notation can be found in [2] and [22]. Throughout the paper, $(R, \mathfrak{m})$ is a Noetherian local ring and $J \subset I$ are two ideals of $R$.

\section{The EQUATIONS OF A STANDARD ALGEBRA AND THE EFFECTIVE RELATIONS}

By a standard $R$-algebra $U$ we mean a graded $R$-algebra $U=\bigoplus_{n>0} U_{n}$, with $U_{0}=R, U=R\left[U_{1}\right]$ and $U_{1}$ minimally generated by $z_{1}, \ldots, z_{s} \in U_{1}$ as an $R$ module. For instance, the $R$-algebra $\mathbf{R}(I)=\bigoplus_{n \geq 0} I^{n}$, the $R / I$-algebra $\mathbf{G}(I)=$ $\bigoplus_{n \geq 0} I^{n} / I^{n+1}$ and the $R / \mathfrak{m}$-algebra $\mathbf{F}(I)=\bigoplus_{n \geq 0} I^{n} / \mathfrak{m} I^{n}$ are standard algebras.

Let $V=R\left[T_{1}, \ldots, T_{s}\right]$ be a polynomial ring with variables $T_{1}, \ldots, T_{s}$ and let $\varphi: V \rightarrow U$ be the induced presentation of $U$ sending $T_{i}$ to $z_{i}$. Let $Q=\bigoplus_{n>1} Q_{n}$ be the kernel of $\varphi$, whose elements will be referred to as the equations of $\bar{U}$. As before, let $Q\langle n\rangle$ be the ideal generated by the homogeneous equations of $U$ of degree at most $n$. The relation type of $U$, denoted by $\operatorname{rt}(U)$, is the least integer $N \geq 1$, such that $Q=Q\langle N\rangle$. Observe that the relation type of $I, \operatorname{rt}(I)$, defined in Section 1, is nothing else but the relation type of its Rees algebra $\mathbf{R}(I)$.

Although $Q$ depends on the presentation $\varphi$, the quotients $(Q / Q\langle n-1\rangle)_{n}=$ $Q_{n} / V_{1} Q_{n-1}, n \geq 2$, do not. Indeed, let $\mathbf{S}\left(U_{1}\right)$ be the symmetric algebra of $U_{1}$ and let $\alpha: \mathbf{S}\left(U_{1}\right) \rightarrow U$ be the canonical morphism induced by the identity in degree one. Given $n \geq 2$, the module of effective $n$-relations of $U$ is defined to be $E(U)_{n}=$ $\operatorname{ker}\left(\alpha_{n}\right) / U_{1} \operatorname{ker}\left(\alpha_{n-1}\right)$. One can prove that, for any $n \geq 2,(Q / Q\langle n-1\rangle)_{n} \cong E(U)_{n}$ (see [20, Definition 2.2]). In particular, the relation type of $U$ can be calculated as the least integer $N \geq 1$, such that $E(U)_{n}=0$ for all $n \geq N+1$.

This description of $(Q / Q\langle n-1\rangle)_{n}$ as $E(U)_{n}=\operatorname{ker}\left(\alpha_{n}\right) / U_{1} \operatorname{ker}\left(\alpha_{n-1}\right)$ has the advantage of being canonical. However, it is often useful to express $(Q / Q\langle n-1\rangle)_{n}$ as a Koszul homology module (see [20, Corollary 2.7]). Concretely,

$$
(Q / Q\langle n-1\rangle)_{n} \cong E(U)_{n} \cong H_{1}(z ; U)_{n},
$$


for $n \geq 2$, where $H_{1}(z ; U)_{n}$ denotes the first homology module of the complex:

$$
\ldots \rightarrow \wedge_{2}\left(R^{s}\right) \otimes U_{n-2} \stackrel{\partial_{2, n-2}}{\longrightarrow} \wedge_{1}\left(R^{s}\right) \otimes U_{n-1} \stackrel{\partial_{1, n-1}}{\longrightarrow} U_{n} \rightarrow 0
$$

where the Koszul differentials are defined as follows: if $e_{1}, \ldots, e_{s}$ stands for the canonical basis of $R^{s}$ and $u \in U_{n-2}$ and $v \in U_{n-1}$, then

$$
\partial_{2, n-2}\left(e_{i} \wedge e_{j} \otimes u\right)=e_{j} \otimes z_{i} \cdot u-e_{i} \otimes z_{j} \cdot u \text { and } \partial_{1, n-1}\left(e_{i} \otimes v\right)=z_{i} \cdot v .
$$

As usual, $Z_{1}(z ; U)$ and $B_{1}(z ; U)$ will stand for the graded modules of 1-cycles and 1-boundaries, respectively, of the Koszul complex of $z$. Observe that $B_{1}(z ; U)_{1}=0$.

For the sake of easy reference we finish this section by stating the following two observations.

Remark 2.1. For $n \geq 2$, there is an isomorphism $\tau_{n}:(Q / Q\langle n-1\rangle)_{n} \cong H_{1}(z ; U)_{n}$ sending the class of $F \in Q_{n}$ modulo $Q\langle n-1\rangle$ to the homology class of the $s$-tuple $\left(F_{1}(z), \ldots, F_{s}(z)\right) \in Z_{1}(z ; U)_{n} \subset U_{n-1} \oplus \ldots \oplus U_{n-1}$, where $F_{1}, \ldots, F_{s}$ are elements in $V_{n-1}$ satisfying $F=T_{1} F_{1}+\ldots+T_{s} F_{s}$. For $n=1$, setting $Q\langle 0\rangle=0$ and taking into account that $B_{1}(z ; U)_{1}=0$, there is an isomorphism $\tau_{1}: Q_{1} \rightarrow Z_{1}(z ; U)_{1}$.

Proof. Take $f=\varphi: V \rightarrow U$ and $g=\mathrm{id}_{V}$ in [20, Theorem 2.4].

Remark 2.2. Let $U$ be a standard $R$-algebra and let $Q=\bigoplus_{n \geq 1} Q_{n}$ be the equations of $U$. Suppose that $Q=Q\langle N\rangle$ for some $N \geq 1$. Set

$$
\mathcal{C}=\left\{F_{1,1}, \ldots, F_{1, s_{1}}, \ldots, F_{N, 1}, \ldots, F_{N, s_{N}}\right\},
$$

with $F_{i, j} \in Q_{i}$. Then $\mathcal{C}$ is a minimal generating set of $Q$ if and only if, for each $n=$ $1, \ldots, N$, the classes of $F_{n, 1}, \ldots, F_{n, s_{n}}$ modulo $Q\langle n-1\rangle$ are a minimal generating set of $(Q / Q\langle n-1\rangle)_{n}$.

\section{The equations of the Rees algebra via Koszul homology}

As always, $(R, \mathfrak{m})$ is a Noetherian local ring and $I$ is an ideal of $R$. The purpose of this section is to describing the modules $(Q / Q\langle n-1\rangle)_{n}$, where $Q$ are the equations of $\mathbf{R}(I)$. By Remark 2.1, this is equivalent to describing the graded components of a Koszul homology module. Although the next lemma is written for $\mathbf{R}(I)$, similar results can also be established for $\mathbf{G}(I)$ or $\mathbf{F}(I)$.

Lemma 3.1. Let $(R, \mathfrak{m})$ be a Noetherian local ring and let $I$ be an ideal of $R$. Let $x_{1}, \ldots, x_{s}, y$ be a minimal generating set of $I$ and $J=\left(x_{1}, \ldots, x_{s}\right)$. Then, for each integer $n \geq 2$, there is a short exact sequence

$$
\begin{aligned}
0 & \rightarrow \frac{H_{1}\left(x_{1} t, \ldots, x_{s} t ; \mathbf{R}(I)\right)_{n}}{y t H_{1}\left(x_{1} t, \ldots, x_{s} t ; \mathbf{R}(I)\right)_{n-1}} \\
& \longrightarrow H_{1}\left(x_{1} t, \ldots, x_{s} t, y t ; \mathbf{R}(I)\right)_{n} \stackrel{\sigma_{n}}{\longrightarrow} \frac{J I^{n-1}: y^{n}}{J I^{n-2}: y^{n-1}} \rightarrow 0 .
\end{aligned}
$$

Moreover, $\sigma_{n}$ sends the class of a cycle $\left(w_{1} t^{n-1}, \ldots, w_{s} t^{n-1}, w_{s+1} t^{n-1}\right), w_{i} \in I^{n-1}$, to the class of $a \in\left(J I^{n-1}: y^{n}\right)$, where $w_{s+1}=a y^{n-1}+b$ for some $b \in J I^{n-2}$. In particular, if $J$ is a reduction of $I$, then $r t(I) \geq r_{j}(I)+1$. 
Proof. Take $z=x_{1} t, \ldots, x_{s} t$ and $\underline{z}=x_{1} t, \ldots, x_{s} t, y t$ in It $\subset \mathbf{R}(I)=R[I t]=$ $\bigoplus_{n \geq 0} I^{n}$. Consider the induced graded long exact sequence of Koszul homology:

$$
\begin{aligned}
& H_{1}(z ; \mathbf{R}(I))_{n-1} \stackrel{\left(\rho_{1}\right)_{n}}{\longrightarrow} H_{1}(z ; \mathbf{R}(I))_{n} \\
\longrightarrow & H_{1}(\underline{z} ; \mathbf{R}(I))_{n} \longrightarrow H_{0}(z ; \mathbf{R}(I))_{n-1} \stackrel{\left(\rho_{0}\right)_{n}}{\longrightarrow} H_{0}(z ; \mathbf{R}(I))_{n},
\end{aligned}
$$

where $\left(\rho_{i}\right)_{n}$ is just the multiplication by $\pm y t$. We get the following short exact sequence:

$$
0 \rightarrow \operatorname{coker}\left(\rho_{1}\right)_{n} \longrightarrow H_{1}(\underline{z} ; \mathbf{R}(I))_{n} \stackrel{\sigma_{n}^{\prime}}{\longrightarrow} \operatorname{ker}\left(\rho_{0}\right)_{n} \rightarrow 0 .
$$

Clearly, $\operatorname{coker}\left(\rho_{1}\right)_{n}=H_{1}(z ; \mathbf{R}(I))_{n} / y t H_{1}(z ; \mathbf{R}(I))_{n-1}$. On the other hand,

$$
H_{0}(z ; \mathbf{R}(I))_{n-1}=I^{n-1} / J I^{n-2} .
$$

Thus $\operatorname{ker}\left(\rho_{0}\right)_{n}=I^{n-1} \cap\left(J I^{n-1}: y\right) / J I^{n-2}$. One can check that $\sigma_{n}^{\prime}$ maps the homology class of a cycle $\left(w_{1} t^{n-1}, \ldots, w_{s} t^{n-1}, w_{s+1} t^{n-1}\right) \in \bigoplus_{i=1}^{s+1} I^{n-1} t^{n-1}$ to the class of $w_{s+1} \in I^{n-1} \cap\left(J I^{n-1}: y\right)$ modulo $J I^{n-2}$. Finally, consider the mapping $\theta_{n}$,

$$
\operatorname{ker}\left(\rho_{0}\right)_{n}=\frac{I^{n-1} \cap\left(J I^{n-1}: y\right)}{J I^{n-2}} \stackrel{\theta_{n}}{\longrightarrow} \frac{J I^{n-1}: y^{n}}{J I^{n-2}: y^{n-1}},
$$

defined as follows: for each $w \in I^{n-1} \cap\left(J I^{n-1}: y\right)$, since $I^{n-1}=y^{n-1} R+J I^{n-2}$, take $a \in R$ and $b \in J I^{n-2}$ such that $w=a y^{n-1}+b$. Clearly, $a \in\left(J I^{n-1}: y^{n}\right)$. Let $\bar{w}$ be the class of $w$ modulo $J I^{n-2}$ and let $\bar{a}$ be the class of $a$ modulo $\left(J I^{n-2}\right.$ : $\left.y^{n-1}\right)$. Set $\theta_{n}(\bar{w})=\bar{a}$. An elementary computation shows that $\theta_{n}$ is a well-defined isomorphism. Take $\sigma_{n}=\theta_{n} \circ \sigma_{n}^{\prime}$.

Although Lemma 3.1 has a version for $n=1$, we are more interested in a kind of analogue obtained from considering $x_{1}, \ldots, x_{s}$ and $x_{1}, \ldots, x_{s}, y$ as sequences of elements of degree zero in $I \subset R$.

Remark 3.2. Let $(R, \mathfrak{m})$ be a Noetherian local ring and let $I$ be an ideal of $R$. Let $x_{1}, \ldots, x_{s}, y$ be a minimal generating set of $I$ and $J=\left(x_{1}, \ldots, x_{s}\right)$. Then there is an exact sequence

$$
0 \rightarrow \frac{H_{1}\left(x_{1}, \ldots, x_{s} ; R\right)}{y H_{1}\left(x_{1}, \ldots, x_{s} ; R\right)} \longrightarrow H_{1}\left(x_{1}, \ldots, x_{s}, y ; R\right) \stackrel{\tilde{\sigma}_{1}}{\longrightarrow} \frac{(J: y)}{J} \rightarrow 0,
$$

where $\tilde{\sigma}_{1}$ sends the homology class of a cycle $\left(w_{1}, \ldots, w_{s}, w_{s+1}\right) \in R^{s+1}$ to the class of $w_{s+1} \in(J: y)$. In particular, if $x_{1}, \ldots, x_{s}$ is an $R$-sequence, $H_{1}\left(x_{1}, \ldots, x_{s} ; R\right)=$ 0 and $\tilde{\sigma}_{1}$ is an isomorphism.

In the next lemma we will prove that, for $n \geq 2, H_{1}\left(x_{1} t, \ldots, x_{s} t ; \mathbf{R}(I)\right)_{n}=0$, provided that $x_{1}, \ldots, x_{s}$ is an $R$-sequence such that $x_{1}^{*}, \ldots, x_{s-1}^{*}$ is a $\mathbf{G}(I)$-sequence. Notice that we are not saying that the whole $H_{1}\left(x_{1} t, \ldots, x_{s} t ; \mathbf{R}(I)\right)$ vanishes. In fact, if $s>1, x_{1}\left(x_{2} t\right) \in x_{1} t \cdot \mathbf{R}(I)$, whereas $x_{1} \notin x_{1} t \cdot \mathbf{R}(I)$; hence $x_{1} t, \ldots, x_{s} t$ is not an $\mathbf{R}(I)$-sequence.

Before this, recall the result of P. Valabrega and G. Valla in [25, Corollary 2.7] which characterizes to be a $\mathbf{G}(I)$-sequence. Let $J \subset I$ be two ideals of a Noetherian local ring $R$. Let $x_{1}, \ldots, x_{s}$ be a minimal generating set of $J$ and write $J_{0}=0$ and $J_{i}=\left(x_{1}, \ldots, x_{i}\right)$, for $i=1, \ldots, s$. In particular, the initial forms $x_{1}^{*}, \ldots, x_{s}^{*}$ in $\mathbf{G}(I)$ are in $I / I^{2}$. Then, $x_{1}^{*}, \ldots, x_{s}^{*}$ is a $\mathbf{G}(I)$-sequence if and only if $x_{1}, \ldots, x_{s}$ is an 
$R$-sequence and the so-called Valabrega-Valla modules $V V_{J_{i}}(I)_{n}=J_{i} \cap I^{n} / J_{i} I^{n-1}$ are zero for all $i=1, \ldots, s$ and all $n \geq 1$.

Lemma 3.3. Let $(R, \mathfrak{m})$ be a Noetherian local ring and let $J \subset I$ be two ideals of $R$. Let $x_{1}, \ldots, x_{s}$ be a minimal generating set of $J$. Write $J_{0}=0$ and $J_{i}=\left(x_{1}, \ldots, x_{i}\right)$, for $i=1, \ldots, s$. Let $n \geq 2$. Then the following two conditions are equivalent:

(i) $H_{1}\left(x_{1} t, \ldots, x_{i} t ; \mathbf{R}(I)\right)_{n}=0$, for all $i=1, \ldots, s$;

(ii) $\left(J_{i-1} I^{n-1}: x_{i}\right) \cap I^{n-1} / J_{i-1} I^{n-2}=0$, for all $i=1, \ldots, s$.

Suppose that, in addition, $x_{1}, \ldots, x_{s}$ is an $R$-sequence and $x_{1}^{*}, \ldots, x_{s-1}^{*}$ is a $\mathbf{G}(I)$ sequence. Then, for all $n \geq 2$ and all $i=1, \ldots, s$,

$$
H_{1}\left(x_{1} t, \ldots, x_{i} t ; \mathbf{R}(I)\right)_{n}=0 .
$$

Proof. For a fixed $n \geq 2$, let us prove the equivalence between $(i)$ and $(i i)$ by induction on $s \geq 1$. If $s=1$, then

$$
H_{1}\left(x_{1} t ; \mathbf{R}(I)\right)_{n} \cong\left(0: x_{1}\right) \cap I^{n-1} \cong\left(J_{0} I^{n-1}: x_{1}\right) \cap I^{n-1} / J_{0} I^{n-2},
$$

and the claim follows.

Now, take $s>1$ and set $z=x_{1} t, \ldots, x_{s-1} t$ and $\underline{z}=x_{1} t, \ldots, x_{s-1} t, x_{s} t$. The induced graded long exact sequence of Koszul homology gives rise to the exact sequence

$$
H_{1}(z ; \mathbf{R}(I))_{n} \longrightarrow H_{1}(\underline{z} ; \mathbf{R}(I))_{n} \longrightarrow H_{0}(z ; \mathbf{R}(I))_{n-1} \stackrel{\left(\rho_{0}\right)_{n}}{\longrightarrow} H_{0}(z ; \mathbf{R}(I))_{n},
$$

where $\left(\rho_{0}\right)_{n}$ is the multiplication by $\pm x_{s} t$. Then

$$
\operatorname{ker}\left(\rho_{0}\right)_{n}=\frac{\left(\left(x_{1} t, \ldots, x_{s-1} t\right): x_{s} t\right)_{n-1}}{\left(x_{1} t, \ldots, x_{s-1} t\right)_{n-1}} \cong \frac{\left(J_{s-1} I^{n-1}: x_{s}\right) \cap I^{n-1}}{J_{s-1} I^{n-2}} .
$$

Therefore, one has the following exact sequence:

$$
H_{1}(z ; \mathbf{R}(I))_{n} \longrightarrow H_{1}(\underline{z} ; \mathbf{R}(I))_{n} \longrightarrow \frac{\left(J_{s-1} I^{n-1}: x_{s}\right) \cap I^{n-1}}{J_{s-1} I^{n-2}} \rightarrow 0 .
$$

By the induction hypothesis, $H_{1}\left(x_{1} t, \ldots, x_{i} t ; \mathbf{R}(I)\right)_{n}=0$, for all $i=1, \ldots, s-1$, is equivalent to $\left(J_{i-1} I^{n-1}: x_{i}\right) \cap I^{n-1} / J_{i-1} I^{n-2}=0$, for all $i=1, \ldots, s-1$. Therefore, using the former exact sequence, one deduces that $H_{1}\left(x_{1} t, \ldots, x_{i} t ; \mathbf{R}(I)\right)_{n}=0$, for all $i=1, \ldots, s$, is equivalent to $\left(J_{i-1} I^{n-1}: x_{i}\right) \cap I^{n-1} / J_{i-1} I^{n-2}=0$, for all $i=1, \ldots, s$.

Suppose that $x_{1}, \ldots, x_{s}$ is an $R$-sequence and $x_{1}^{*}, \ldots, x_{s-1}^{*}$ is a $\mathbf{G}(I)$-sequence. Let us see that $\left(J_{i-1} I^{n-1}: x_{i}\right) \cap I^{n-1} / J_{i-1} I^{n-2}=0$, for all $i=1, \ldots, s$ and all $n \geq 2$. Using the aforementioned result of P. Valabrega and G. Valla,

$$
V V_{J_{i}}(I)_{n-1}=J_{i} \cap I^{n-1} / J_{i} I^{n-2}=0
$$

for all $i=1, \ldots, s-1$ and all $n \geq 2$. Since $x_{1}, \ldots, x_{s}$ is an $R$-sequence, then $\left(J_{i-1}: x_{i}\right)=J_{i-1}$ for all $i=1, \ldots, s$. Therefore, for all $i=1, \ldots, s$ and all $n \geq 2$, $J_{i-1} I^{n-2} \subseteq\left(J_{i-1} I^{n-1}: x_{i}\right) \cap I^{n-1} \subseteq\left(J_{i-1}: x_{i}\right) \cap I^{n-1}=J_{i-1} \cap I^{n-1}=J_{i-1} I^{n-2}$.

One can state a different version of the second part of Lemma 3.3. which will in turn lead to a slightly different version of the main result of this paper (see Remark 4.3).

Recall that $x_{1}, \ldots, x_{s}$ is a $d$-sequence if $\left(J_{i}: x_{i+1} x_{j}\right)=\left(J_{i}: x_{j}\right)$ for all $0 \leq i \leq$ $s-1$ and all $j \geq i+1$ (where $J_{0}=0, J_{i}=\left(x_{1}, \ldots, x_{i}\right)$ and $J_{s}=J$, as before). 
This condition is equivalent to $\left(J_{i}: x_{i+1}\right) \cap J=J_{i}$ for all $0 \leq i \leq s-1$. Clearly, $R$-sequences are $d$-sequences.

Remark 3.4. Let $(R, \mathfrak{m})$ be a Noetherian local ring and let $I$ be an ideal of $R$. Let $x_{1}, \ldots, x_{s}$ be a minimal generating set of $J$, where $J=\left(x_{1}, \ldots, x_{s}\right)$ is a reduction of $I$ with reduction number $r=\mathrm{r}_{J}(I)$. Assume that

(a) $x_{1}, \ldots, x_{s}$ is a $d$-sequence and

(b) $V V_{J_{i}}(I)_{r+1}=\left(x_{1}, \ldots, x_{i}\right) \cap I^{r+1} /\left(x_{1}, \ldots, x_{i}\right) I^{r}=0$ for all $i=1, \ldots, s-1$.

Then $H_{1}\left(x_{1} t, \ldots, x_{i} t ; \mathbf{R}(I)\right)_{n}=0$, for all $n \geq r+2$ and all $i=1, \ldots, s$. Suppose that, in addition,

(c) $x_{1}, \ldots, x_{s}$ is an $R$-sequence and

(d) $V V_{J_{i}}(I)_{r}=\left(x_{1}, \ldots, x_{i}\right) \cap I^{r} /\left(x_{1}, \ldots, x_{i}\right) I^{r-1}=0$ for all $i=1, \ldots, s-1$.

Then $H_{1}\left(x_{1} t, \ldots, x_{i} t ; \mathbf{R}(I)\right)_{r+1}=0$, for all $i=1, \ldots, s$.

Proof. Since $J$ is a reduction of $I$ with reduction number $r, I^{r+1}=J I^{r}$. Using $(a)$ and $(b)$, for $i=1, \ldots, s$, we get

$$
\left(J_{i-1}: x_{i}\right) \cap I^{r+1}=\left(J_{i-1}: x_{i}\right) \cap J \cap I^{r+1}=J_{i-1} \cap I^{r+1}=J_{i-1} I^{r} .
$$

Therefore, for all $i=1, \ldots, s,\left(J_{i-1}: x_{i}\right) \cap I^{r+1}=J_{i-1} I^{r}$. Now, using the result of N.V. Trung in [24, Proposition 4.7(i)], one has $\left(J_{i-1}: x_{i}\right) \cap I^{n}=J_{i-1} I^{n-1}$, for all $n \geq r+1$ and all $i=1, \ldots, s$. This clearly implies that

$$
\left(J_{i-1} I^{n}: x_{i}\right) \cap I^{n}=J_{i-1} I^{n-1},
$$

for all $n \geq r+1$ and all $i=1, \ldots, s$, which by Lemma 3.3. is equivalent to $H_{1}\left(x_{1} t, \ldots, x_{i} t ; \mathbf{R}(I)\right)_{n}=0$, for all $n \geq r+2$ and all $i=1, \ldots, s$.

Now suppose that $(c)$ and $(d)$ hold. Then, for all $i=1, \ldots, s$,

$$
J_{i-1} I^{r-1} \subseteq\left(J_{i-1} I^{r}: x_{i}\right) \cap I^{r} \subseteq\left(J_{i-1}: x_{i}\right) \cap I^{r}=J_{i-1} \cap I^{r}=J_{i-1} I^{r-1} .
$$

Therefore, for all $i=1, \ldots, s,\left(J_{i-1} I^{r}: x_{i}\right) \cap I^{r}=J_{i-1} I^{r-1}$. By Lemma 3.3. $H_{1}\left(x_{1} t, \ldots, x_{i} t ; \mathbf{R}(I)\right)_{r+1}=0$, for all $i=1, \ldots, s$.

\section{MAin RESUlt}

We now have all the ingredients to prove the main result of the paper. As always, set $V=R\left[X_{1}, \ldots, X_{s}, Y\right]$ and let $Q$ be the kernel of the polynomial presentation $\varphi: V \rightarrow \mathbf{R}(I)$ sending $X_{i}$ to $x_{i} t$ and $Y$ to $y t$.

Theorem 4.1. Let $(R, \mathfrak{m})$ be a Noetherian local ring and let $I$ be an ideal of $R$. Let $x_{1}, \ldots, x_{s}, y$ be a minimal generating set of $I$ and $J=\left(x_{1}, \ldots, x_{s}\right)$. Assume that $x_{1}, \ldots, x_{s}$ is an $R$-sequence and that $x_{1}^{*}, \ldots, x_{s-1}^{*}$ is a $\mathbf{G}(I)$-sequence. Then, for each $n \geq 2$, the map sending $F \in Q_{n}$ to $F(0, \ldots, 0,1) \in\left(J I^{n-1}: y^{n}\right)$ induces an isomorphism of $R$-modules

$$
\left[\frac{Q}{Q\langle n-1\rangle}\right]_{n} \cong \frac{J I^{n-1}: y^{n}}{J I^{n-2}: y^{n-1}}
$$

In particular, if $J$ is a reduction of $I$ with reduction number $r=r_{J}(I)$, then $r t(I)=$ $r_{J}(I)+1$ and there is a form $Y^{r+1}-\sum X_{i} F_{i} \in Q_{r+1}$, with $F_{i} \in V_{r}$, such that $Q=\left(Y^{r+1}-\sum X_{i} F_{i}\right)+Q\langle r\rangle$. 
Proof. By Lemma 3.1 with $z=x_{1} t, \ldots, x_{s} t$ and $\underline{z}=x_{1} t, \ldots, x_{s} t, y t$,

$$
0 \rightarrow \frac{H_{1}(z ; \mathbf{R}(I))_{n}}{y t H_{1}(z ; \mathbf{R}(I))_{n-1}} \longrightarrow H_{1}(\underline{z} ; \mathbf{R}(I))_{n} \stackrel{\sigma_{n}}{\longrightarrow} \frac{J I^{n-1}: y^{n}}{J I^{n-2}: y^{n-1}} \rightarrow 0
$$

is an exact sequence for all $n \geq 2$. By Lemma 3.3. $H_{1}(z ; \mathbf{R}(I))_{n}=0$ for all $n \geq 2$. Therefore $H_{1}(\underline{z} ; \mathbf{R}(I))_{n} \stackrel{\sigma_{n}}{\cong}\left(J I^{n-1}: y^{n}\right) /\left(J I^{n-2}: y^{n-1}\right)$, for $n \geq 2$. With Remark 2.1, we conclude:

$$
\left[\frac{Q}{Q\langle n-1\rangle}\right]_{n} \stackrel{\tau_{n}}{\cong} H_{1}(\underline{z} ; \mathbf{R}(I))_{n} \stackrel{\sigma_{n}}{\cong} \frac{J I^{n-1}: y^{n}}{J I^{n-2}: y^{n-1}},
$$

for all $n \geq 2$. Given $F \in Q_{n}$, write $F=\sum_{i=1}^{s} X_{i} F_{i}+Y G$, with $F_{i}, G \in$ $V_{n-1}$. Then the morphism $\tau_{n}$ sends the class of $F$ to the homology class of $\left(F_{1}(\underline{z}), \ldots, F_{s}(\underline{z}), G(\underline{z})\right)$. But $G(\underline{z})=G\left(x_{1}, \ldots, x_{s}, y\right) t^{n-1}$ and $G\left(x_{1}, \ldots, x_{s}, y\right)=$ $G(0, \ldots, 0,1) y^{n-1}+b$, for some $b \in J I^{n-2}$. By Lemma 3.1, $\sigma_{n}$ sends the homology class of $\left(F_{1}(\underline{z}), \ldots, F_{s}(\underline{z}), G(\underline{z})\right)$ to the class of $G(0, \ldots, 0,1)$ modulo $\left(J I^{n-2}: y^{n-1}\right)$ and notice that $G(0, \ldots, 0,1)=F(0, \ldots, 0,1)$.

If $J$ is a reduction of $I$ with reduction number $r,\left(J I^{n-1}: y^{n}\right)=R$ for all $n \geq r+1$. Therefore $(Q / Q\langle n-1\rangle)_{n}=0$ for all $n>r+1$ and $(Q / Q\langle r\rangle)_{r+1} \cong$ $R /\left(J I^{r-1}: y^{r}\right) \neq 0$, since $y^{r} \notin J I^{r-1}$. Therefore $\operatorname{rt}(I)=r+1$. Finally, notice that the containment $y^{r+1} \in J I^{r}$ induces an equation of the form $Y^{r+1}-\sum_{i} X_{i} F_{i}$, with $F_{i} \in V_{r}$, which is sent by $\sigma_{n} \circ \tau_{n}$ to the class of 1 in $R /\left(J I^{r-1}: y^{r}\right)$.

Remark 4.2. In Theorem 4.1, we use the hypotheses " $x_{1}, \ldots, x_{s}$ is an $R$-sequence and $x_{1}^{*}, \ldots, x_{s-1}^{*}$ is a $\mathbf{G}(I)$-sequence" to assure $H_{1}\left(x_{1} t, \ldots, x_{s} t ; \mathbf{R}(I)\right)_{n}=0$, for all $n \geq 2$, by means of Lemma 3.3. Therefore, in Theorem 4.1, one can substitute, if needed, the hypotheses " $x_{1}, \ldots, x_{s}$ is an $R$-sequence and $x_{1}^{*}, \ldots, x_{s-1}^{*}$ is a $\mathbf{G}(I)$ sequence" by the weaker hypotheses " $\left(J_{i-1} I^{n-1}: x_{i}\right) \cap I^{n-1} / J_{i-1} I^{n-2}=0$, for all $i=1, \ldots, s$ and all $n \geq 2$ ". See Example 5.2 as an application of this comment.

With the weaker hypotheses pinpointed in Remark 3.4, we get the next version of Theorem 4.1 .

Remark 4.3. Let $(R, \mathfrak{m})$ be a Noetherian local ring and let $I$ be an ideal of $R$. Let $x_{1}, \ldots, x_{s}, y$ be a minimal generating set of $I$, where $J=\left(x_{1}, \ldots, x_{s}\right)$ is a reduction of $I$ with reduction number $r=\mathrm{r}_{J}(I)$. Assume that

(a) $x_{1}, \ldots, x_{s}$ is a $d$-sequence and

(b) $V V_{J_{i}}(I)_{r+1}=\left(x_{1}, \ldots, x_{i}\right) \cap I^{r+1} /\left(x_{1}, \ldots, x_{i}\right) I^{r}=0$ for all $i=1, \ldots, s-1$.

Then $\operatorname{rt}(I)=\mathrm{r}_{J}(I)+1$. Suppose that, in addition,

(c) $x_{1}, \ldots, x_{s}$ is an $R$-sequence and

(d) $V V_{J_{i}}(I)_{r}=\left(x_{1}, \ldots, x_{i}\right) \cap I^{r} /\left(x_{1}, \ldots, x_{i}\right) I^{r-1}=0$ for all $i=1, \ldots, s-1$.

Then there is a form $Y^{r+1}-\sum X_{i} F_{i} \in Q_{r+1}$, with $F_{i} \in V_{r}$, such that $Q=$ $\left(Y^{r+1}-\sum X_{i} F_{i}\right)+Q\langle r\rangle$.

Proof. This follows from Lemma 3.1 and the proof of Theorem 4.1 but using Remark 3.4 instead of Lemma 3.3 . 
Discussion 4.4. The hypotheses of Remark 4.3 are connected with the work of S. Huckaba in [13, Theorem 1.4] and N.V. Trung in [24, Theorem 6.4] (see also [3, Theorem 3.2], [12, [5, Theorem 5.3], 23]). In [13, Theorems 1.4, 1.5], S. Huckaba proved that if $I$ is an ideal with $l(I)=$ height $(I)+1 \geq 2$ and such that any minimal reduction $J$ of $I$ can be generated by a $d$-sequence $x_{1}, \ldots, x_{s}$ with $x_{1}^{*}, \ldots, x_{s-1}^{*}$ being a $\mathbf{G}(I)$-sequence $(s=l(I))$, then $\operatorname{rt}(I) \leq \mathrm{r}_{J}(I)+1$. If in addition $\mu(I)=l(I)+1$, then the equality $\operatorname{rt}(I)=\mathrm{r}_{J}(I)+1$ holds. In particular, $r=\mathrm{r}_{J}(I)$ is independent of $J$. In fact, N.V. Trung improved this last result in [24, Theorem 6.4] by showing that $r$ coincides with the Castelnuovo-Mumford regularity of $\mathbf{R}(I)$. To prove $\operatorname{rt}(I) \geq$ $\mathrm{r}_{J}(I)+1$, S. Huckaba showed that the equality $I^{r+1}=J I^{r}$ induces an equation of $\mathbf{R}(I)$ of maximum degree. In Theorem 4.1 and with a different approach, we have completed the description of the whole ideal of equations of $\mathbf{R}(I)$.

As a corollary of Theorem 4.1, one can prove the result of W.V. Vasconcelos in [26. Theorem 2.3.3] for any reduction number not necessarily equal to 1.

Corollary 4.5. Let $(R, \mathfrak{m})$ be a Cohen-Macaulay local ring of dimension $d$. Let $I$ be an $\mathfrak{m}$-primary ideal of $R$ minimally generated by $x_{1}, \ldots, x_{d}, y$, where $J=$ $\left(x_{1}, \ldots, x_{d}\right)$ is a reduction of $R$ with reduction number $r=r_{J}(I)$. Suppose that $x_{1}^{*}, \ldots, x_{d-1}^{*}$ is a $\mathbf{G}(I)$-sequence. Then there is a form $Y^{r+1}-\sum X_{i} F_{i} \in Q_{r+1}$, with $F_{i} \in V_{r}$, such that $Q=\left(Y^{r+1}-\sum X_{i} F_{i}\right)+Q\langle r\rangle$. In particular, $r t(I)=r+1$.

Proof. Since $R$ is Cohen-Macaulay and $I$ is $\mathfrak{m}$-primary, $x_{1}, \ldots, x_{d}$ is an $R$-sequence and the results follow from Theorem 4.1 .

Before stating the next result, recall that for an ideal $L$ of $R$ and any standard $R$-algebra $U$, the relation type of $U \otimes R / L$ is $\operatorname{rt}(U \otimes R / L) \leq \operatorname{rt}(U)$ (see e.g. [20, Example 3.2]). Hence $\operatorname{rt}(\mathbf{F}(I)) \leq \operatorname{rt}(\mathbf{G}(I)) \leq \operatorname{rt}(I)$. In fact, for any $n \geq 2$ and writing $E(I)_{n}$ for $E(\mathbf{R}(I))_{n}$ (see Section 2 to recall the definition of effective relations), there is an exact sequence $E(I)_{n+1} \rightarrow E(I)_{n} \rightarrow E(\mathbf{G}(I))_{n} \rightarrow 0$. In particular, $\operatorname{rt}(I)=\operatorname{rt}(\mathbf{G}(I))$ and $E(I)_{N} \cong E(\mathbf{G}(I))_{N}$ for $N=\operatorname{rt}(I)$ (20, Proposition 3.3]; see also [8, p. 268]). In our context, $\operatorname{rt}(\mathbf{F}(I))$ is also equal to $\operatorname{rt}(I)$, which is not a general fact (see the Conjecture of G. Valla in [9, §2] and a counterexample in [21, Example 4.4]; see also [8, p. 268 and Corollary 2.6]).

As another corollary of Theorem 4.1, we recover the following result of W. Heinzer and M.-K. Kim in [7, Theorem 5.6].

Corollary 4.6. Let $(R, \mathfrak{m})$ be a Noetherian local ring with infinite residue field $k=R / \mathfrak{m}$ and let $I$ be an ideal of $R$. Let $x_{1}, \ldots, x_{s}, y$ be a minimal generating set of $I$, where $J=\left(x_{1}, \ldots, x_{s}\right)$ is a reduction of $I$ with reduction number $r=r_{J}(I)$. Assume that $x_{1}, \ldots, x_{s}$ is an $R$-sequence and that $x_{1}^{*}, \ldots, x_{s-1}^{*}$ is a $\mathbf{G}(I)$-sequence. Then there is a form $Y^{r+1}-\sum X_{i} F_{i}$, with $F_{i} \in k\left[X_{1}, \ldots, X_{s}, Y\right]$ forms of degree $r$ and $\mathbf{F}(I) \cong k\left[X_{1}, \ldots, X_{s}, Y\right] /\left(Y^{r+1}-\sum X_{i} F_{i}\right)$. In particular, $r t(\mathbf{F}(I))=r_{J}(I)+$ $1=\operatorname{rt}(I)$.

Proof. By Theorem 4.1, $\operatorname{rt}(\mathbf{F}(I)) \leq \operatorname{rt}(I)=r+1$. By [7, Lemma 5.2], $E(\mathbf{F}(I))_{n}=0$ for all $2 \leq n \leq r$ and $E(\mathbf{F}(I))_{r+1} \neq 0$. Thus $\mathbf{F}(I)$ has only equations of degree $r+1$ and $\operatorname{rt}(\mathbf{F}(I))=r+1$. By Theorem 4.1. $E(I)_{r+1}$ is cyclic and generated by the equation of $\mathbf{R}(I)$ induced by the containment $y^{r+1} \in J I^{r}$. Therefore the same happens with $E(\mathbf{F}(I))_{r+1}$ (see [5, Proposition 3.2] or [8, p. 268]). 


\section{Some examples}

Our purpose is to take advantage of Theorem 4.1 to obtain a minimal generating set of the equations of $\mathbf{R}(I)$. The ascending chain of colon ideals $\left\{\left(J I^{n-1}: y^{n}\right)\right\}_{n \geq 1}$, which need not be rigid but which stabilize in $R$, can be calculated in any computer algebra system, giving a possibly alternative procedure to finding the equations of $\mathbf{R}(I)$.

The following ideal $I$, for a specific $p \geq 1$, is often used as an example of an ideal of relation type at least $p$. However, we are not aware of any reference with a detailed description of a minimal generating set of the equations of $\mathbf{R}(I)$, for a general $p \geq 1$. As was said above, Theorem 4.1 will be crucial to our purposes.

Example 5.1. Let $(R, \mathfrak{m})$ be a Noetherian local ring. Let $a_{1}, a_{2}$ be an $R$-sequence and $p \geq 2$. Set $x_{1}=a_{1}^{p}, x_{2}=a_{2}^{p}$ and $y=a_{1} a_{2}^{p-1}$. Let $I$ be the ideal generated by $x_{1}, x_{2}, y$. Set $V=R\left[X_{1}, X_{2}, Y\right]$ and let $\varphi: V \rightarrow \mathbf{R}(I)$ be the presentation of $\mathbf{R}(I)$ sending $X_{i}$ to $x_{i} t$ and $Y$ to $y t$. Then a minimal generating set of the ideal $Q=\operatorname{ker}(\varphi)$ is obtained from:

- a unique equation $F_{n}\left(X_{1}, X_{2}, Y\right)=a_{1}^{p-n} Y^{n}-a_{2}^{p-n} X_{1} X_{2}^{n-1} \in Q_{n}$ of degree $n$, for each $n, 2 \leq n \leq p$;

- two equations $F_{1}\left(X_{1}, X_{2} ; Y\right)=a_{1}^{p-1} Y-a_{2}^{p-1} X_{1}$ and $G_{1}\left(X_{1}, X_{2}, Y\right)=$ $a_{2} Y-a_{1} X_{2} \in Q_{1}$ of degree 1 .

Proof. We start by proving the hypotheses in Theorem 4.1. Clearly $x_{1}, x_{2}$ is an $R$ sequence and $J=\left(x_{1}, x_{2}\right)$ is a reduction of $I$ since $I^{p}=J I^{p-1}$. By [18, Corollary 3], a monomial $m$ on $a_{1}, a_{2}$ belongs to an ideal generated by monomials $m_{1}, \ldots, m_{r}$ on $a_{1}, a_{2}$ if and only if $m$ is a multiple of some $m_{i}$. It follows that $y^{p-1} \notin J I^{p-2}$ and $I^{p-1} \nsubseteq J I^{p-2}$. Thus $\mathrm{r}_{J}(I)=p-1$.

Claim. $x_{1}^{*}$ is a $\mathbf{G}(I)$-sequence. By [25, Corollary 2.7], it suffices to prove

$$
V V_{\left(x_{1}\right)}(I)_{n}=x_{1} R \cap I^{n} / x_{1} I^{n-1}=0
$$

for all $n \geq 1$. Fix $n \geq 1$. By [18, Proposition 1],

$$
x_{1} R \cap I^{n}=\left(L_{i, j, k} \mid i, j, k \text { positive integers such that } i+j+k=n\right),
$$

where $L_{i, j, k}=\operatorname{lcm}\left(a_{1}^{p}, a_{1}^{i p} a_{2}^{j p}\left(a_{1} a_{2}^{p-1}\right)^{k}\right)$. Let us prove that $L_{i, j, k}$ is in $x_{1} I^{n-1}$.

Indeed, if $i \geq 1$, then $L_{i, j, k}=a_{1}^{i p} a_{2}^{j p}\left(a_{1} a_{2}^{p-1}\right)^{k}=a_{1}^{p}\left[a_{1}^{(i-1) p} a_{2}^{j p}\left(a_{1} a_{2}^{p-1}\right)^{k}\right] \in$ $x_{1} I^{n-1}$ and we have finished. Hence we can suppose $i=0$ and $j+k=n$. If $k=0$, then $j=n$ and $L_{0, j, 0}=a_{1}^{p} a_{2}^{j p} \in x_{1} I^{n-1}$. Suppose $0<k \leq p$. Then $L_{0, j, k}=a_{1}^{p} a_{2}^{j p+k(p-1)}=a_{1}^{p}\left(a_{2}^{p}\right)^{j+k-1} a_{2}^{p-k} \in x_{1} I^{n-1}$. Finally, if $k>p$, then $L_{0, j, k}=a_{2}^{j p}\left(a_{1} a_{2}^{p-1}\right)^{k}=a_{1}^{p}\left[a_{1}^{k-p} a_{2}^{j p+k(p-1)}\right]=a_{1}^{p}\left[a_{1}^{k-p} a_{2}^{(k-p)(p-1)} a_{2}^{j p+p(p-1)}\right]=$ $a_{1}^{p}\left(a_{2}^{p}\right)^{j+p-1}\left(a_{1} a_{2}^{p-1}\right)^{k-p} \in x_{1} I^{n-1}$.

Note that if $p>2$, then $x_{2}^{*}$ is not a $\mathbf{G}(I)$-sequence because

$$
\left(a_{1}^{p+2} a_{2}^{p-2}\right) x_{2}=a_{1}^{p}\left(a_{1} a_{2}^{p-1}\right)^{2}=x_{1} y^{2} \in I^{3},
$$

where $\left(a_{1}^{p+2} a_{2}^{p-2}\right) \in I \backslash I^{2}$.

Therefore, we can apply Theorem 4.1 and deduce that, for all $n \geq 2$,

$$
\left[\frac{Q}{Q\langle n-1\rangle}\right]_{n} \cong \frac{J I^{n-1}: y^{n}}{J I^{n-2}: y^{n-1}} .
$$

In particular, since $\left(J I^{p-2}: y^{p-1}\right) \subsetneq\left(J I^{p-1}: y^{p}\right)=R$, then $\operatorname{rt}(I)=\mathrm{r}_{J}(I)+1$. 
Claim. $\left(J I^{n-1}: y^{n}\right)=\left(a_{1}^{p-n}, a_{2}\right)$ for $2 \leq n \leq p-1$. First note that, for all $2 \leq$ $n \leq p-1, a_{2} \in\left(J I^{n-1}: y^{n}\right)$ since $a_{2} y^{n}=a_{1}^{n} a_{2}^{n(p-1)+1}=a_{1} a_{2}^{p}\left(a_{1}^{n-1} a_{2}^{(n-1)(p-1)}\right)=$ $a_{1} a_{2}^{p}\left(a_{1} a_{2}^{p-1}\right)^{n-1} \in J I^{n-1}$. Since $\left(J I^{n-1}: y^{n}\right)$ is generated by monomials on $a_{1}, a_{2}$, and $a_{2} \in\left(J I^{n-1}: y^{n}\right)$, there is only one possible remaining generator: a power of $a_{1}$. Since $a_{1}^{p-n} y^{n}=a_{1}^{p-n} a_{1}^{n} a_{2}^{n(p-1)}=a_{1}^{p} a_{2}^{n(p-1)}=a_{2}^{p-n} a_{1}^{p}\left(a_{2}^{p}\right)^{n-1} \in J I^{n-1}$, then $a_{1}^{p-n} \in\left(J I^{n-1}: y^{n}\right)$. However, and again using [18, Corollary 3], one has $a_{1}^{p-n-1} \notin\left(J I^{n-1}: y^{n}\right)$.

Hence $(Q / Q\langle p-1\rangle)_{p} \cong\left(J I^{p-1}: y^{p}\right) /\left(J I^{p-2}: y^{p-1}\right)=R /\left(a_{1}, a_{2}\right)$ and, for $2 \leq n \leq p-1$,

$$
\left[\frac{Q}{Q\langle n-1\rangle}\right]_{n} \cong \frac{J I^{n-1}: y^{n}}{J I^{n-2}: y^{n-1}}=\frac{\left(a_{1}^{p-n}, a_{2}\right)}{\left(a_{1}^{p-n+1}, a_{2}\right)} \cong \frac{\left(a_{1}^{p-n}\right)}{\left(a_{1}^{p-n+1}, a_{1}^{p-n} a_{2}\right)} .
$$

In other words, for each $2 \leq n \leq p,(Q / Q\langle n-1\rangle)_{n}$ is generated by a single element that corresponds to the class of $a_{1}^{p-n}$ ( 1 if $\left.n=p\right)$. To find this element, consider the identity $a_{1}^{p-n} y^{n}=a_{2}^{p-n} a_{1}^{p}\left(a_{2}^{p}\right)^{n-1}$, which induces the equation $F_{n}\left(X_{1}, X_{2}, Y\right)=$ $a_{1}^{p-n} Y^{n}-a_{2}^{p-n} X_{1} X_{2}^{n-1} \in Q_{n}$. Since the isomorphism of Theorem 4.1 sends the class of $F_{n}$ to the class of $F_{n}(0,0,1)=a_{1}^{p-n}$, we are done. By Remark 2.2 $F_{n}$ is in a minimal generating set of $Q$, for $2 \leq n \leq p$.

To finish, let us find the equations of degree one. Though this is trivial, we sketch the proof here to show the similarity with the greater degrees. As before, one shows that $J=\left(a_{1}^{p-1}, a_{2}\right)$. Using Remark 3.2 ,

$$
H_{1}\left(x_{1}, x_{2}, y ; R\right) \stackrel{\tilde{\sigma}_{1}}{\cong} \frac{(J: y)}{J}=\frac{\left(a_{1}^{p-1}, a_{2}\right)}{\left(a_{1}^{p}, a_{2}^{p}\right)}
$$

Identify $Q_{1}$ with $Z_{1}\left(x_{1}, x_{2}, y ; R\right)$ and $B_{1}=\left\langle x_{1} Y-y X_{1}, x_{2} Y-y X_{2}, x_{1} X_{2}-x_{2} X_{1}\right\rangle$ with $B_{1}\left(x_{1}, x_{2}, y ; R\right)$. Then $Q_{1} / B_{1}$ is minimally generated by the classes of the two equations corresponding to the classes of $a_{1}^{p-1}$ and $a_{2}$. The identities $a_{1}^{p-1} y=$ $a_{2}^{p-1} a_{1}^{p}$ and $a_{2} y=a_{1} a_{2}^{p}$ induce the desired equations $F_{1}\left(X_{1}, X_{2}, Y\right)=a_{1}^{p-1} Y-$ $a_{2}^{p-1} X_{1}$ and $G_{1}\left(X_{1}, X_{2}, Y\right)=a_{2} Y-a_{1} X_{2} \in Q_{1}$, since by $\tilde{\sigma}_{1}$ their classes are sent to the classes of $F_{1}(0,0,1)=a_{1}^{p-1}$ and $G_{1}(0,0,1)=a_{2}$. Clearly, $B_{1} \subset\left\langle F_{1}, G_{1}\right\rangle$ and $F_{1}, G_{1}$ constitute a minimal generating set of $Q_{1}$.

In the next example, although the initial forms $x_{1}^{*}, x_{2}^{*}$ are zero-divisors in $\mathbf{G}(I)$, where $I=\left(x_{1}, x_{2}, y\right)$, one may still apply Theorem 4.1 because the conditions of Lemma 3.3 " $\left(J_{i-1} I^{n-1}: x_{i}\right) \cap I^{n-1} / J_{i-1} I^{n-2}=0$, for all $i=1, \ldots, s$, for all $n \geq 2$ ", still hold (see Remark 4.2).

Example 5.2. Let $(R, \mathfrak{m})$ be a Noetherian local ring. Let $a_{1}, a_{2}$ be an $R$-sequence and let $p \geq 5$ be an odd integer. Set $x_{1}=a_{1}^{p}, x_{2}=a_{2}^{p}$ and $y=a_{1}^{2} a_{2}^{p-2}$. Let $I$ be the ideal generated by $x_{1}, x_{2}, y$ and set $J=\left(x_{1}, x_{2}\right)$. Then $J$ is a reduction of $I$ with reduction number $\mathrm{r}_{J}(I)=p-1$ and $\left(x_{1} I^{n-1}: x_{2}\right) \cap I^{n-1} / x_{1} I^{n-2}=0$, for all $n \geq 2$. However, the initial forms $x_{1}^{*}, x_{2}^{*}$ are zero-divisors in $\mathbf{G}(I)$. Applying Remark 4.2, one obtains the minimal generating set of the equations of $\mathbf{R}(I)$ :

- an equation $F_{n}\left(X_{1}, X_{2}, Y\right)=Y^{p}-X_{1}^{2} X_{2}^{p-2}$ of degree $p$;

- an equation $F_{n}\left(X_{1}, X_{2}, Y\right)=a_{2} Y^{n}-a_{1} X_{1} X_{2}^{n-1}$ of degree $n=(p+1) / 2$; 
- an equation $F_{n}\left(X_{1}, X_{2}, Y\right)=a_{1}^{p-2 n} Y^{n}-a_{2}^{p-2 n} X_{1} X_{2}^{n-1}$ of degree $n$, for each $n, 2 \leq n \leq(p-1) / 2$;

- two equations $F_{1}\left(X_{1}, X_{2} ; Y\right)=a_{1}^{p-2} Y-a_{2}^{p-2} X_{1}$ and $G_{1}\left(X_{1}, X_{2}, Y\right)=$ $a_{2}^{2} Y-a_{1}^{2} X_{2}$ of degree 1 .

We leave the details to the reader.

The next example shows that the weaker hypotheses in Remark 4.3 are not enough to assure that there is only one equation of $\mathbf{R}(I)$ of maximum degree.

Example 5.3. Let $k$ be a field and $R=k[X, Y]_{(X, Y)} /\left(X Y, Y^{2}\right)_{(X, Y)}$. Let $x$ and $y$ be the classes of $X$ and $Y$ in $R$. Let $\mathfrak{m}=(x, y)$ be the maximal ideal of $R$. Then the relation type of $\mathfrak{m}$ is 2 and there are two equations of degree 2 in a minimal generating set of equations of $\mathbf{R}(\mathfrak{m})$.

Proof. Set $J=(x)$. Since $y^{2}=0 \in J \mathfrak{m}$ and $y \notin J$, then $\mathfrak{m}^{2}=J \mathfrak{m}$ and $J$ is a reduction of $\mathfrak{m}$ with reduction number 1 . Moreover, since $(0: x)=\left(0: x^{2}\right), x$ is a $d$-sequence. By Remark [4.3, $\operatorname{rt}(\mathfrak{m}) \leq \mathrm{r}_{J}(\mathfrak{m})+1=2$. Set $V=k[S, T]$ and let $\psi: V \rightarrow \mathbf{G}(\mathfrak{m})$ be the presentation of $\mathbf{G}(\mathfrak{m})$ sending $S$ to $x+\mathfrak{m}^{2}$ and $T$ to $y+\mathfrak{m}^{2}$. For $n \geq 2, \mathfrak{m}^{n}=\left(x^{n}\right)$. Thus $\mathfrak{m}^{2} / \mathfrak{m}^{3}$ is a $k$-vector space of dimension 1 . Therefore $\operatorname{ker}\left(\psi_{2}\right) \subset V_{2}$ must have dimension 2. In fact, $\operatorname{ker}\left(\psi_{2}\right)=\left\langle S T, T^{2}\right\rangle$. Since $\operatorname{ker}\left(\psi_{1}\right)=0$, then $E(\mathbf{G}(\mathfrak{m}))_{2}$ is minimally generated by two elements. We finish by using that $E(\mathfrak{m})_{2} \cong E(\mathbf{G}(\mathfrak{m}))_{2}([20$, Proposition 3.3]).

The next example shows that if the deviation $\mu(I)$ - height $(I)$ fails to be equal to 1 , then there might be several equations of $\mathbf{R}(I)$ of maximum degree.

Example 5.4. Let $(R, \mathfrak{m})$ be a two-dimensional regular local ring, $p \geq 2$. Then $\operatorname{rt}\left(\mathfrak{m}^{p}\right)=2$ and there are $\left(\begin{array}{l}p \\ 2\end{array}\right)$ equations of degree 2 in a minimal generating set of equations of $\mathbf{R}\left(\mathfrak{m}^{p}\right)$.

Proof. Let $x, y$ be a regular system of parameters of $R, V=R[X, Y]$ and let $\varphi: V \rightarrow \mathbf{R}(\mathfrak{m})$ be the presentation of $\mathbf{R}(\mathfrak{m})$ sending $X$ to $x t$ and $Y$ to $y t$. Since $x, y$ is an $R$-sequence, $\operatorname{ker}(\varphi)=\langle x Y-y X\rangle$. Set $V(p)=R\left[X^{p}, X^{p-1} Y, \ldots, X Y^{p-1}, Y^{p}\right]$ and let $\varphi(p): V(p) \rightarrow \mathbf{R}\left(\mathfrak{m}^{p}\right)$ be the $p$-th Veronese transform of $\varphi$. Note that $\operatorname{ker}\left(\varphi(p)_{n}\right)=\operatorname{ker}\left(\varphi_{p n}\right)=\langle x Y-y X\rangle V_{p n-1}$.

Set $W=R\left[T_{0}, T_{1}, \ldots, T_{p}\right]$ and let $\psi: W \rightarrow V(p)$ be the polynomial presentation of $V(p)$ sending $T_{i}$ to $X^{p-i} Y^{i}$. It is known (see e.g. [17, Proposition 2.5]) that the kernel of $\psi$ is the determinantal ideal generated by the $2 \times 2$ minors of the matrix $\mathcal{M}$, where $\mathcal{M}$ is

$$
\left(\begin{array}{cccc}
T_{0} & T_{1} & \ldots & T_{p-1} \\
T_{1} & T_{2} & \ldots & T_{p}
\end{array}\right)
$$

In particular, $\operatorname{ker}(\psi)=\operatorname{ker}(\psi)\langle 2\rangle$.

Consider $\Phi=\varphi(p) \circ \psi: R\left[T_{0}, T_{1}, \ldots, T_{p}\right] \rightarrow \mathbf{R}\left(\mathfrak{m}^{p}\right)$, the polynomial presentation of $\mathbf{R}\left(\mathfrak{m}^{p}\right)$ sending $T_{i}$ to $x^{i} y^{p-i}$ and let $Q=\operatorname{ker}(\Phi)$ be the ideal of equations of $\mathbf{R}\left(\mathfrak{m}^{p}\right)$. Let us see that $Q_{n}=\operatorname{ker}\left(\psi_{n}\right)+W_{n-1} Q_{1}$, for all $n \geq 2$. Indeed, given $F \in Q_{n}$, since $\operatorname{ker}\left(\varphi(p)_{n}\right)=\langle x Y-y X\rangle V_{p n-1}$, one can find $F_{i} \in W_{n-1}$ and $G_{i} \in Q_{1}$ such that $\psi_{n}(F)=\psi_{n}\left(\sum F_{i} G_{i}\right)$. Therefore, $\left(F-\sum F_{i} G_{i}\right) \in \operatorname{ker}\left(\psi_{n}\right)$ and $F \in$ $\operatorname{ker}\left(\psi_{n}\right)+W_{n-1} Q_{1}$.

Since $\operatorname{ker}(\psi)=\operatorname{ker}(\psi)\langle 2\rangle$, then $Q_{n}=\operatorname{ker}\left(\psi_{n}\right)+W_{n-1} Q_{1}=W_{n-2} \operatorname{ker}\left(\psi_{2}\right)+$ $W_{n-1} Q_{1} \subseteq W_{n-2} Q_{2} \subseteq Q_{n}$, for all $n \geq 2$. Therefore $Q=Q\langle 2\rangle, \operatorname{rt}\left(\mathfrak{m}^{n}\right)=2$ and $\mu\left(Q_{2} / W_{1} Q_{1}\right) \leq\left(\begin{array}{l}p \\ 2\end{array}\right)$. 
Let us prove that $Q_{2} / W_{1} Q_{1}$ is minimally generated by $\left(\begin{array}{c}p \\ 2\end{array}\right)$ elements, which are precisely the classes of the $2 \times 2$ minors of $\mathcal{M}$. Since $Q_{1} \subset \mathfrak{m} W_{1}$, then $W_{1} Q_{1} \subset \mathfrak{m} W_{2}$. Setting $L=\operatorname{ker}\left(\psi_{2}\right), M=W_{2}$ and $N=V(p)_{2}$, we have

$$
\frac{Q_{2}}{W_{1} Q_{1}} \otimes k \cong \frac{Q_{2}}{\mathfrak{m} Q_{2}+W_{1} Q_{1}}=\frac{\operatorname{ker}\left(\psi_{2}\right)+W_{1} Q_{1}}{\mathfrak{m} \operatorname{ker}\left(\psi_{2}\right)+W_{1} Q_{1}} \cong \frac{L}{\mathfrak{m} L+\left(L \cap W_{1} Q_{1}\right)} .
$$

On the other hand, there is a natural epimorphism

$$
\frac{L}{\mathfrak{m} L+\left(L \cap W_{1} Q_{1}\right)} \rightarrow \frac{L}{L \cap \mathfrak{m} M},
$$

where $L /(L \cap \mathfrak{m} M) \cong(L+\mathfrak{m} M) / \mathfrak{m} M=\operatorname{ker}\left(\psi_{2} \otimes 1_{k}\right)$. Hence,

$$
\mu\left(\frac{Q_{2}}{W_{1} Q_{1}}\right) \geq \operatorname{dim} \frac{L}{(L \cap \mathfrak{m} M)}=\operatorname{dim} \operatorname{ker}\left(\psi_{2} \otimes 1_{k}\right),
$$

which clearly is $\left(\begin{array}{l}p \\ 2\end{array}\right)$. Therefore $\mu\left((Q / Q\langle 1\rangle)_{2}\right)=\left(\begin{array}{l}p \\ 2\end{array}\right)$ and, by Remark 2.2, there are $\left(\begin{array}{l}p \\ 2\end{array}\right)$ equations of degree 2 in a minimal generating set of equations of $\mathbf{R}\left(\mathrm{m}^{p}\right)$.

Note that $J=\left(x_{1}^{p}, x_{2}^{p}\right)$ is a reduction of $\mathfrak{m}^{p}$ with reduction number $\mathrm{r}_{J}\left(\mathfrak{m}^{p}\right)=1$, that $x_{1}^{p}, x_{2}^{p}$ is an $R$-sequence and that $\left(x_{1}^{p}\right)^{*}$ is a $\mathbf{G}\left(\mathfrak{m}^{p}\right)$-sequence.

\section{REFERENCES}

1. I.M. Aberbach, L. Ghezzi, H.T. Hà, Homology multipliers and the relation type of parameter ideals, Pacific J. Math. 226 (2006), 1-40. MR2247853(2007f:13004)

2. W. Bruns, J. Herzog, Cohen-Macaulay rings, Cambridge Studies in Advanced Mathematics, 39. Cambridge University Press, 1993. MR1251956 (95h:13020)

3. T. Cortadellas, S. Zarzuela, On the depth of the fiber cone of filtrations. J. Algebra 198 (1997), 428-445. MR1489906 (98k:13005)

4. E.G. Davis, Ideals of the principal class, $R$-sequences and a certain monoidal transformation, Pacific J. Math. 20 (1967), 197-205. MR0206035 (34:5860)

5. J.M. Giral, F. Planas-Vilanova, Integral degree of a ring, reduction numbers and uniform Artin-Rees numbers, J. Algebra 319 (2008), 3398-3418. MR2408325 (2009g:13012)

6. G.-M. Greuel, G. Pfister, H. Schönemann. Singular. A computer algebra system for polynomial computations. University of Kaiserslautern. http://www.singular.uni-kl.de.

7. W. Heinzer, M.-K. Kim, Properties of the fiber cone of ideals in local rings, Comm. Algebra 31 (2003), 3529-3546. MR1990289 (2004e:13006)

8. W. Heinzer, M.-K. Kim, B. Ulrich, The Gorenstein and complete intersection properties of associated graded rings. J. Pure Appl. Algebra 201 (2005), 264-283. MR2158759 (2006d:13003)

9. M. Herrmann, B. Moonen, O. Villamayor, Ideals of linear type and some variants. The Curves Seminar at Queen's, Vol. VI (Kingston, ON, 1989), Exp. No. H, 37 pp., Queen's Papers in Pure and Appl. Math., 83, Queen's Univ., Kingston, ON, 1989. MR1036038 (91b:13010)

10. J. Hong, A. Simis, W.V. Vasconcelos, On the homology of two-dimensional elimination, J. Symbolic Comput. 43 (2008), no. 4, 275-292. MR.2402032(2009b:14024)

11. J. Hong, A. Simis, W.V. Vasconcelos, The equations of almost complete intersections, arXiv:0906.1591

12. S. Huckaba, Reduction numbers for ideals of higher analytic spread, Math. Proc. Cambridge Philos. Soc. 102 (1987), 49-57. MR886434 (89b:13023)

13. S. Huckaba, On complete $d$-sequences and the defining ideals of Rees algebras, Math. Proc. Cambridge Philos. Soc. 106 (1989), 445-458. MR1010369 (90m:13012)

14. S. Huckaba, C. Huneke, Powers of ideals having small analytic deviation, Amer. J. Math. 114 (1992), 367-403. MR 1156570 (93g:13002)

15. S. Huckaba, C. Huneke, Rees algebras of ideals having small analytic deviation, Trans. Amer. Math. Soc. 339 (1993), 373-402. MR1123455 (93k:13008)

16. C. Huneke, Tight closure and its applications, CBMS Lecture Notes, 88. American Mathematical Society, Providence, RI, 1996. MR.1377268 (96m:13001)

17. B. Johnston, D. Katz, On the relation type of large powers of an ideal, Mathematika 41 (1994), 209-214. MR1288065 (95f:13003) 
18. K. Kiyek, J. Stückrad, Integral closure of monomial ideals on regular sequences. Rev. Mat. Iberoamericana 19 (2003), no. 2, 483-508. MR2023197 (2005d:13014)

19. Y.H. Lai, On the relation type of systems of parameters, J. Algebra 175 (1995), 339-358. MR1338982(96i:13026)

20. F. Planas-Vilanova, On the module of effective relations of a standard algebra. Math. Proc. Cambridge Philos. Soc. 124 (1998), 215-229. MR.1631099 (99h:13002)

21. A. Simis, B. Ulrich, W.V. Vasconcelos, Jacobian dual fibrations. Amer. J. Math. 115 (1993), no. 1, 47-75. MR1209234(94d:13018)

22. I. Swanson, C. Huneke, Integral closure of ideals, rings, and modules. London Mathematical Society Lecture Note Series, 336. Cambridge University Press, Cambridge, 2006. MR 2266432 (2008m:13013)

23. N. V. Trung, Reduction exponent and degree bound for the defining equations of graded rings. Proc. Amer. Math. Soc. 101 (1987), no. 2, 229-236. MR.902533 (89i:13031)

24. N. V. Trung, The Castelnuovo regularity of the Rees algebra and the associated graded ring, Trans. Amer. Math. Soc. 350 (1998), 2813-2832. MR.1473456 (98j:13006)

25. P. Valabrega, G. Valla, Form rings and regular sequences. Nagoya Math. J. 72 (1978), 93-101. MR514892 (80d:14010)

26. W. V. Vasconcelos, On the equations of Rees algebras. J. Reine Angew. Math. 418 (1991), 189-218. MR1111206 (92c:13006)

27. W. V. Vasconcelos and Berndt Ulrich, The equations of Rees algebras of ideals with linear presentation. Math. Z. 214 (1993), no. 1, 79-92. MR.1234599 (94g:13002)

28. H. J. Wang, The relation-type conjecture holds for rings with finite local cohomology, Comm. Algebra 25 (1997), 785-801. MR1433435 (98d:13017)

Departament de Matemàtica Aplicada 1, Universitat Politècnica de Catalunya, Diagonal 647, ETSEIB, 08028 BARCELONA, Spain

E-mail address: ferranmuinos@gmail.com

Departament de Matemàtica Aplicada 1, Universitat Politècnica de Catalunya, Diagonal 647, ETSEIB, 08028 Barcelona, Spain

E-mail address: francesc.planas@upc.edu 